\title{
Targeted Microlesions Reveal Novel Organization of the Hamster Suprachiasmatic Nucleus
}

\author{
Lance J. Kriegsfeld, ${ }^{1}$ Joseph LeSauter, ${ }^{2}$ and Rae Silver ${ }^{1,2,3}$ \\ Departments of Psychology, ${ }^{1}$ Columbia University and ${ }^{2}$ Barnard College, New York, New York 10027, and ${ }^{3}$ Department of Anatomy and Cell Biology, \\ College of Physicians and Surgeons, New York, New York 10032
}

The role of the suprachiasmatic nuclei (SCN) in generating circadian rhythms in physiology and behavior is well established. Recent evidence based on clock gene expression indicates that the rodent SCN are composed of at least two functional subdivisions. In Syrian hamsters (Mesocricetus auratus), cells in a subregion of the caudal $\mathrm{SCN}$ marked by calbindin- $\mathrm{D}_{28 \mathrm{~K}}(\mathrm{CalB})$ express light-induced, but not rhythmic, clock genes (Per1, Per2, and Per3). In the SCN region marked by vasopressinergic cells and fibers, clock gene expression is rhythmic. Importantly, lesions of the CalB subregion that spare a significant portion of the SCN abolish rhythms in locomotor behavior. One possibility is that the CalB subregion is required to maintain SCN function necessary to support all behavioral and physiological rhythms. Alternatively, this subregion may control circadian rhythms in locomotor behavior, whereas other circadian responses in physiology and behavior are sustained by different SCN compartments. The present study sought to distinguish between these possibilities by examining the role of the CalB subregion in a battery of rhythms within an individual animal. The results indicate that lesions of the CalB subregion of the SCN abolish circadian rhythms in behavior (locomotion, drinking, gnawing), physiology (body temperature, heart rate), and hormone secretion (melatonin, cortisol), even when other SCN compartments are spared. Together, these findings suggest a novel fundamental property of SCN organization, with a subset of cells being critical for the maintenance of SCN function manifest in circadian rhythms in physiology and behavior.

Key words: circadian; neuropeptide; pacemaker; suprachiasmatic; clock; diurnal

\section{Introduction}

Virtually all behavioral and physiological processes exhibit circadian cycles with a period of $\sim 24 \mathrm{hr}$. The role of the suprachiasmatic nucleus (SCN) in generating these circadian rhythms in physiology and behavior is well established (Moore and Eichler, 1972; Stephan and Zucker, 1972; Lehman et al., 1987; Ralph et al., 1990). Over the last decade, enormous progress has been made in uncovering the mechanisms responsible for circadian oscillations at the cellular and molecular levels (for review, see Okamura et al., 2002; Panda et al., 2002). To understand rhythm generation in multicellular organisms, it is important to investigate how the molecular mechanisms translate to network properties at the tissue level and to the behavior of the whole organism.

The rodent SCN are composed of $\sim 10,000$ cells in each nucleus (van den Pol and Tsujimoto, 1985; Moore et al., 2002). At the tissue level, the $\mathrm{SCN}$ of rats and mice have been characterized into "core" (ventrolateral) and "shell" (dorsomedial) subregions based on the pattern of neural inputs and outputs as well as in the organization of phenotypically distinct neuronal cell types (Moore, 1996; Moga and Moore, 1997; Moore et al., 2002). Re-

Received Dec. 2, 2003; revised Jan. 14, 2004; accepted Jan. 16, 2004

This work was supported by National Institutes of Health Grants MH-12408 (L.J.K.) and NS-37919 (R.S.). We thank Sarah Marks, Charles Yackulic, and Ruslan Korets for technical assistance.

Correspondence should be addressed to Dr. Rae Silver, Department of Psychology, Columbia University, 1190 Amsterdam Avenue, New York, NY 10027. E-mail: qr@columbia.edu.

DOl:10.1523/JNEUROSCI.5323-03.2004

Copyright $\odot 2004$ Society for Neuroscience $\quad$ 0270-6474/04/242449-09\$15.00/0 cently, we have characterized the hamster (Hamada et al., 2001) and mouse (Karatsoreos et al., 2004) SCN as having two functionally distinct regions, one exhibiting light-induced Period (Per) expression and a nonoverlapping region expressing Per rhythmically (see below). In view of this complex topographical and functional organization, it is interesting to note that the SCN has historically been considered to be a functionally homogenous structure. For example, lesion studies suggest that survival of only a small fraction $(\sim 25 \%)$ of any portion of the $\mathrm{SCN}$ is sufficient to support rhythmic function (Rusak, 1977; Van den Pol and Powley, 1979; Davis and Gorski, 1988; Harrington et al., 1993; Welsh et al., 1995). More recent work on clock gene expression and electrical activity in neurochemically defined SCN subregions suggest that the SCN is not functionally homogenous but instead that different subregions serve different functions (Hamada et al., 2001; Nakamura et al., 2001; Jobst and Allen, 2002).

Syrian hamsters represent an ideal animal model to explore questions of SCN organization, because functional subdivisions have been well-characterized by their chemoarchitecture, providing convenient markers. Our laboratory has shown that lesions bilaterally destroying a small portion of the $\mathrm{SCN}$ marked by calbindin- $\mathrm{D}_{28 \mathrm{~K}}(\mathrm{CalB})$, while sparing up to $67 \%$ of the remainder of the SCN, abolish rhythms in locomotor behavior (LeSauter and Silver, 1999). Per1 and Per2 are light induced, but not endogenously rhythmic, in this CalB subregion. In contrast, the SCN shell, marked by vasopressin-containing cells, has rhythmic Per1, Per2, and Per3 (Hamada et al., 2001). One hypothesis that may 
account for these findings proposes that cells in the CalB region are necessary to coordinate independent oscillator cells in the SCN shell to prevent these cells from drifting out of synchrony (Antle et al., 2003). Following from this hypothesis, lesions destroying the CalB portion of the SCN, while sparing other SCN compartments, should abolish all circadian rhythms in physiology and behavior. Alternatively, the CalB subregion may represent a subdivision uniquely important for arousal measured by locomotor rhythm generation or maintenance, and distinct neurochemical subregions of the SCN may be associated with different rhythmic responses. Distinguishing between these competing hypotheses is critical for establishing SCN functional organization.

\section{Materials and Methods}

Animals and housing. Eighty-three adult male LVG hamsters (Mesocricetus auratus) were used in the present experiment. Seventy-six animals were lesioned, whereas seven intact animals were used to obtain serum for normal cortisol and melatonin patterns (see below). Animals were purchased from Charles River (Wilmington, MA) at 4-5 weeks of age. All animals were housed in translucent propylene cages $(48 \times 27 \times 20 \mathrm{~cm})$ and provided with ad libitum access to food and water for the duration of the study. Cages were equipped with running wheels $(17 \mathrm{~cm}$ diameter) connected to a computer (Dataquest; Datasciences International, St. Paul, MN). Animals were maintained in a colony room with a $24 \mathrm{hr}$ light/dark cycle $\left(14 / 10 \mathrm{hr}\right.$ light/dark) at $23 \pm 1^{\circ} \mathrm{C}$. At least 1 week before the start of the experiment, animals were transferred to cages equipped with running wheels (see below) and housed in constant darkness (DD). All experimental protocols conformed to the Institutional Animal Care and Use Committee guidelines of Columbia University.

Lesions. After 1-2 weeks in DD, 76 male hamsters (110-120 gm) were anesthetized deeply with $60 \mathrm{mg} / \mathrm{kg}$ ketamine and $5 \mathrm{mg} / \mathrm{kg}$ xylazine. The head of the animal was shaved and positioned in a stereotaxic apparatus (David Kopf Intruments, Tujanga, CA), and the animal was prepared for aseptic surgery. Lesions were aimed at the following coordinates: $0.8 \mathrm{~mm}$ anterior to bregma, $0.1 \mathrm{~mm}$ lateral to midline, and $7.9 \mathrm{~mm}$ below the dura. Bilateral electrolytic lesions were made using a Grass Instruments (Quincy, MA) LM-5 lesion maker and stainless steel electrodes insulated with Epoxylite (The Epoxylite Corp., Irvine, CA), excluding the tip (0.20 $\mathrm{mm})$. Current was passed for $10 \mathrm{sec}$ at $0.55 \mathrm{~mA}$.

General methodology and caveats. For the purposes of comparison, records from a single animal with an intact $\mathrm{SCN}$ have been depicted for all measures. The data for all behavioral and physiological measures were collected sequentially from all animals. As a result, minor differences in the period of each rhythm may be observed within an individual animal. However, these differences are consistent with changes in the circadian period that are commonly observed over time. All rhythms measured simultaneously exhibited equivalent periods.

Locomotor behavior. One week before and at least 3 weeks after lesions, locomotor behavior was measured in all animals using cages equipped with running wheels $(17 \mathrm{~cm}$ diameter) connected to a computer (Dataquest; Datasciences International). Cumulative counts were recorded every $10 \mathrm{~min}$.

Drinking behavior. After the lesions, drinking behavior was monitored continuously by placing a wire grid on the bottom of the cage connected to the ground of a circuit closure. The positive lead of the sensor was attached to the spout of the water bottle. Every time the hamster drank from the spout, the circuit was closed, and a count was recorded. Cummulative counts were transmitted to a computer (Dataquest) and recorded every $10 \mathrm{~min}$.

Gnawing behavior. After measures of locomotor and drinking behavior, gnawing rhythms were measured as described previously (LeSauter and Silver, 1994). Eighteen animals showing no disruptions in rhythmic locomotor and drinking behavior, suggesting a missed lesion, were eliminated from further study. For all other animals, a wooden stick was attached to the cage top with a clamp holder. A switch mechanism was mounted on the top of the clamp and to the side of the cage. Gnawing on the stick caused the clamp to move vertically, deflecting the wire attached to the clamp into the wire attached to the side of the cage, thereby closing the circuit. Switch closures were recorded continuously, and cumulative counts were sent to a computer (Dataquest) every $10 \mathrm{~min}$ for a minimum of 3 weeks.

Monitoring of heart rate and body temperature. After the monitoring of gnawing behavior, physiological transmitters (TATTCTA-F40; Datasciences International) were surgically implanted into animals that were deeply anesthetized with $60 \mathrm{mg} / \mathrm{kg}$ ketamine and $5 \mathrm{mg} / \mathrm{kg}$ xylazine. The ventral surface of the abdomen and the thorax to the area of the axilla, slightly cranial on the right side, was shaved. The shaved area was swabbed with Betadine. A midline abdominal skin incision $1 \mathrm{~cm}$ below the diaphragm and no $>2 \mathrm{~cm}$ in length was made. The abdomen was opened by making a $2 \mathrm{~cm}$ incision along the white line of fascia where the abdominal muscles join on the midline. The body of the transmitter was slipped into the abdominal cavity along the sagittal plane and dorsal to the digestive organs so that both leads were oriented toward the head of the animal. For heart rate measurement, the heart leads were threaded through two small holes in the external oblique muscle of the abdominal wall to the right and left of the incision. For negative lead attachment, using a trochar and sleeve, the lead was passed under the skin to an incision made near the clavicle. Similarly, for positive lead placement, the lead was passed under the skin to an incision made cranial to the last rib. The leads were then secured against the chest muscles by attaching a suture just behind the tabs, and the abdominal opening and the smaller two lead access points were closed. After surgery, the animals were returned to their cages. The animals were allowed 1 week to recover after surgery. To monitor heart rate and body temperature, the animals' cages were placed on an RA-1010 receiver plate (Datasciences International) connected to a computer. The plates were situated so that the hamster was always within range of the receiver plate (even when rearing). Body temperature and heart rate were transmitted and monitored continuously. Mean body temperature and heart rate were recorded every $5 \mathrm{~min}$ using Datasciences ART software (Datasciences International).

Gonadal measurements. At the time that transmitters were implanted (8-10 weeks after exposure to DD), the scrotum was dampened with alcohol, and the length and width of the left testis was measured to the nearest $0.1 \mathrm{~mm}$ with calipers. The estimated testis volume (ETV) was calculated as: $\mathrm{ETV}=$ testis width ${ }^{2} \times$ testis length.

Blood sampling. After heart rate and body temperature measurements, animals were implanted with a jugular catheter for future blood sampling. An additional seven control animals not receiving surgery or prior monitoring were also catheterized to obtain standard hormone values. Animals were anesthetized with $60 \mathrm{mg} / \mathrm{kg}$ ketamine and $5 \mathrm{mg} / \mathrm{kg}$ xylazine, and a catheter made out of SILASTIC tubing (inner diameter, 0.025 inch; outer diameter, 0.047 inch; Dow-Corning, Midland, MI) was inserted into the right jugular vein. The free end of the catheter was passed under the skin and exited near the base of the skull. The catheter was filled with heparinized saline $(10 \mathrm{U} / \mathrm{ml})$ and connected to a stainless steel tube (26 gauge) that was sealed with a piece of SILASTIC tubing that was closed at one end. The end of the tube was embedded in dental cement for protection and anchored into the skull with four screws. The animals were allowed $5 \mathrm{~d}$ to recover before blood sampling began. For animals that displayed rhythmic behavior, blood sampling began at a random circadian time for each animal (circadian time 1, 4, 7, 10, 13, 16, 19, and 22) to control for effects of prior sampling on later samples. For arrhythmic animals, blood sampling began at a random local time and continued every $3 \mathrm{hr}$ thereafter for $24 \mathrm{hr}$. To collect blood samples from freely moving animals, $12-15$ inches of PE 50 tubing was attached to the tube on the end of the catheter. A syringe was attached to the end of the tubing, and a $0.3-0.4 \mathrm{ml}$ sample of blood was withdrawn. An equal amount of saline was replaced after each sample. Samples were centrifuged at 3000 $\mathrm{rpm}$, and serum was separated. Serum samples were stored at $-80^{\circ} \mathrm{C}$ until assayed.

Hormone assays. Enzyme immunoassay (EIA) kits were used for measuring serum levels of cortisol and melatonin (Alpco Diagnostics, Windham, NH). Melatonin was measured after extraction by reversephase column chromatography. The cortisol EIA did not require an extraction procedure. The samples were assayed in duplicate according to 
the manufacturer's instructions. The sensitivity of the cortisol assay was $8.4 \mathrm{nmol} / \mathrm{ml}$, whereas the sensitivity for melatonin was $0.34 \mathrm{pg} / \mathrm{ml}$. The intra-assay variability for the melatonin and cortisol assays was 5.6 and $3.9 \%$, respectively. Both assays were validated in the present study for use in hamsters by assessing parallelism with the standard curve in serially diluted pooled hamster serum. All samples were run in single assay (one for cortisol and one for melatonin) in duplicate.

Perfusion and histology. One day after blood sample collection, hamsters were anesthetized deeply with sodium pentobarbital $(200 \mathrm{mg} / \mathrm{kg})$ and perfused intracardially with $150 \mathrm{ml}$ of $0.9 \%$ saline, followed by $300-$ $400 \mathrm{ml}$ of $4 \%$ paraformaldehyde in $0.1 \mathrm{~m} \mathrm{PBS}, \mathrm{pH}$ 7.3. Brains were postfixed for $2-3 \mathrm{hr}$ at $4^{\circ} \mathrm{C}$ and cryoprotected in $20 \%$ sucrose in $0.1 \mathrm{~m}$ PBS overnight. Coronal sections were cut on a cryostat and processed as free-floating sections. Every fourth section was stained for vasopressin or vasoactive intestinal polypeptide (VIP), whereas every other section was stained for CalB. Polyclonal antibodies raised against vasopressin and VIP (Incstar, Stillwater, MN) were diluted at 1:10,000. The monoclonal CalB antibody (Sigma, St. Louis, MO) was used at 1:20,000. Antisera were detected using either biotinylated goat anti-rabbit (vasopressin and VIP) or biotinylated horse anti-mouse secondaries (Vector Laboratories, Burlingame, CA) and an avidin-biotin-HRP conjugate (Vector Laboratories) with $\mathrm{DAB}$ (Polysciences, Warrington, PA) as the chromogen. Sections were mounted onto glass slides, dehydrated in a graded series of ethanols, and cleared in xylenes, and coverslips were applied.

Microscopy and analysis of the SCN. To assess the extent of the lesions, we used vasopressin and VIP as markers for distinct SCN subregions. The presence of vasopressin-immunoreactive (-ir) and VIP-ir neurons in adjacent sections of the SCN was taken as evidence of surviving SCN tissue. Whether or not CalB-ir cells remained after the lesion was assessed as described previously (LeSauter and Silver, 1999). Images of brain sections were captured using a CCD video camera (XC77; Sony, Tokyo, Japan) attached to an Olympus BH-2 microscope (Olympus, Melville, $\mathrm{NY}$ ). The extent of SCN tissue surviving ablation was assessed using NIH Image and measuring the remaining SCN tissue in sections stained with peptidergic markers. Total SCN size averaged over two intact hamsters of the same age and body mass was used as a point of comparison to estimate the percentage of SCN tissue remaining. Restated, surviving SCN tissue was measured in lesioned animals, and the percentage of SCN tissue remaining was calculated as: percentage of surviving tissue $=($ twodimensional area of surviving tissue/two-dimensional area of intact $\mathrm{SCN}) \times 100$.

Delineation of SCN subregions. Various terms have been used to demarcate distinct SCN subregions. Here, we have defined subregions based on chemoarchitecture. For example, we used the descriptive terms "CalB subregion" and "vasopressin subregion" to delineate nonoverlapping regions of the SCN, recognizing that these regions contain other cell phenotypes. Because putative functional characteristics of the hamster SCN map onto neurochemical organization (Hamada et al., 2001, 2003), we felt that this type of analysis was most appropriate for the present investigation. Additional analysis will be required to determine how hamster SCN regions characterized by their clock gene expression correspond to those previously classified as "core" and "shell" in studies of rats and mice (Moore, 1996; Abrahamson and Moore, 2001; Moore et al., 2002)

Analyses and statistics. The power of all rhythms was assessed using a Fourier analysis (Dataquest or Clocklab programs). An animal was considered rhythmic when the highest peak occurred at $\sim 1$ cycle/d, with an absolute power of at least $0.005 \mathrm{mV} / \mathrm{Hz}$. For hormonal data, rhythms from individual animals were considered rhythmic if two consecutive sample values were greater than 2 SDs above the mean nadir values of unlesioned control animals.

For animals that maintained rhythmic function after the lesions, a Pearson Product Moment correlation was performed between the power of the rhythm and a qualitative measure of vasopressin, VIP, and CalB surviving ablation. The extent of sparing of cells and fibers was estimated as follows: as noted above, every fourth section was immunostained for vasopressin and VIP, whereas alternate sections were stained for CalB. Each of these sections were examined by an investigator blind to the experimental condition of the animal and scored on a scale of $1-5$, in
Table 1. Peptidergic analysis of animals with partial SCN lesions indicates that CalB is necessary for behavioral (locomotor, drinking, and gnawing) circadian rhythms

\begin{tabular}{lllllll}
\hline Number & AVP & VIP & CalB & Locomotor & Drinking & Gnawing \\
\hline 11 & + & + & - & - & - & - \\
7 & - & + & + & + & + & + \\
5 & - & + & - & - & - & - \\
$17^{a}$ & + & + & + & + & + & + \\
$16^{b}$ & + & + & + & + & + & + \\
2 & - & - & - & - & - & - \\
\hline
\end{tabular}

+ , Presence of peptide or behavioral rhythm; - , absence of peptide or behavioral rhythm; AVP, arginine vasopressin. ${ }^{a}$ Lesion missed SCN.

${ }^{b}$ Partial lesion of SCN.

which 1 represents $<25 \%$ of the intact nucleus, 5 represents an intact $\mathrm{SCN}$, and a unilateral lesion is scored as 3 .

\section{Results}

\section{Lesion assessment and behavioral analysis}

In 41 of 58 animals investigated for all behavioral and physiological measures, the SCN were partially $(n=39)$ or fully $(n=2)$ ablated on both sides of the brain (summarized in Table 1). Seventeen hamsters had lesions that missed the SCN, and these animals displayed rhythmic locomotor, drinking, and gnawing behavior (Fig. 1). Of the 39 partially lesioned animals, 23 animals with sparing of one (or both) CalB subnuclei remained rhythmic in all behaviors (locomotor, drinking, and gnawing) (Fig. 2). Animals of most interest in the present study were 16 animals with both CalB nuclei destroyed that did not exhibit a circadian rhythm in all behavioral measures (Fig. 2). Not surprisingly, the two animals with complete SCN lesions were arrhythmic in all behavioral measures.

The extent of SCN damage for partially lesioned animals was variable (summarized in Table 1) and is pictured for two individuals (whose behavior is shown in Fig. 2) in Figure 3. One animal with a lesion missing the SCN is also shown to illustrate the normal distribution of the SCN peptides measured. In one behaviorally arrhythmic animal shown, hamster 21-4, the CalB subregion was ablated in both SCN with sparing of VIP and vasopressin in adjacent sections (sparing $~ 55 \%$ of the SCN). Another animal shown, hamster 41-1, exhibited a larger bilateral SCN lesion (sparing $\sim 40 \%$ of the SCN) but, importantly, sparing a portion of the CalB subregion unilaterally (behaviorally rhythmic).

Of the 16 animals that were behaviorally arrhythmic, no surviving CalB cells were seen in the SCN. The brains of 11 of these hamsters with an ablated CalB subregion exhibited VIP and vasopressin cell and fiber staining in adjacent SCN sections, whereas the SCN of five animals contained only VIP. For animals with partial lesions sparing a portion of the CalB subregion, 16 animals had both vasopressin and VIP cells and fibers in adjacent sections, whereas seven animals had VIP but not vasopressin remaining. All animals with partial SCN lesions that were behaviorally rhythmic had spared CalB cells in the SCN (Table 1).

\section{Body temperature and heart rate}

Thirteen animals with lesions missing the SCN had circadian rhythms in body temperature and heart rate. All 15 animals with partial lesions sparing a portion of the CalB subregion also exhibited rhythms in body temperature and heart rate. Thirteen animals with lesions destroying the CalB subregion (while sparing either vasopressin, VIP, or both) did not display rhythms in body temperature or heart rate. Animals with complete SCN lesions $(n=2)$ did not exhibit a circadian pattern in either body temper- 

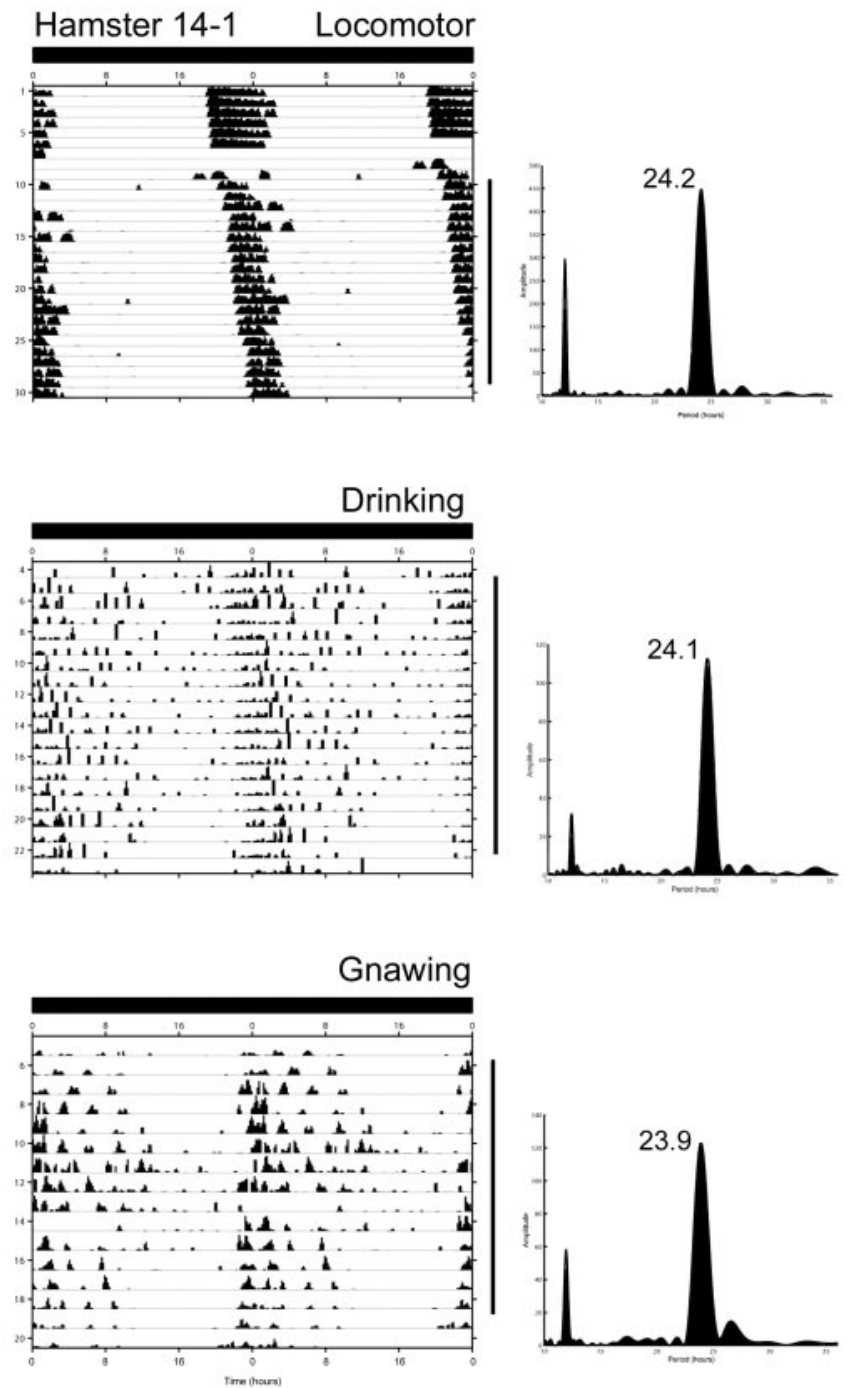

Figure 1. Actograms depicting locomotor (top), drinking (middle), and gnawing (bottom) behavior of hamster 14-1, an animal with a missed SCN lesion. Spectral analyses are presented to the right of each actogram. Black vertical bars to the right of the actograms indicate the days on which the analyses were performed.

ature or heart rate. Because of transmitter failures, body temperature and heart rate rhythms were not obtained from four animals with missed lesions, three animals with partial lesions sparing CalB, and three animals with partial lesions eliminating CalB. Representative records are shown for an animal with a lesion missing the SCN (Fig. 4) and animals with lesions of the SCN either sparing or eliminating CalB (Fig. 5).

\section{Gonadal measures}

As an indirect measure of melatonin secretion, gonadal measures were recorded after extended exposure to DD(Fig. 6). Virtually all animals ( $n=16$ of 17 ) with missed lesions exhibited regressed gonads, indicative of a functional melatonin rhythm. Animals with partial SCN lesions destroying the entire CalB subregion $(n=16)$ or complete SCN lesions $(n=2)$ all had nonregressed gonads, significantly larger than hamsters with missed lesions $(p<0.05)$. Animals with partial lesions sparing portions of the CalB nucleus $(n=18)$ exhibited variability in gonadal size, ranging from nonregressed to regressed.

\section{Melatonin and cortisol}

Serum samples from seven randomly selected animals with partial SCN lesions bilaterally ablating the CalB subregion, with significant sparing of vasopressin, VIP, or both, were assayed for melatonin and cortisol. Serum from six animals with partial lesions, sparing some CalB, was also selected randomly to be assayed. Finally, samples from seven unlesioned control animals were assayed for both hormones to use as a point of comparison for normal cortisol and melatonin rhythms and values in Syrian hamsters (see Materials and Methods). Results are summarized in Table 2. Four of the six animals with partial SCN lesions sparing a portion of the CalB nucleus exhibited rhythms in both melatonin and cortisol, one showed rhythmic cortisol but not melatonin, and the last was rhythmic in neither melatonin nor cortisol. Six of the seven animals with partial SCN lesions abolishing CalB were arrhythmic in melatonin and cortisol, whereas one animal showed a rhythm in both hormones. Figure 7 depicts the rhythms of cortisol and melatonin in an unlesioned control animal. Figures of representative melatonin and cortisol rhythms from animals with (hamster 21-4) and without (hamster 41-1) elimination of the CalB subregion are shown in Figure 8.

\section{Rhythm amplitude and SCN neurochemistry}

In all animals maintaining rhythmicity after partial SCN lesions, all behavioral measures (wheel running, drinking, and gnawing) were significantly positively correlated $(p<0.01)$ with the amount of sparing of CalB (Table 3 ). The higher the score assigned for sparing of CalB after SCN microlesions, the greater the amplitude of circadian rhythms. The amplitude of all behavioral rhythms was not significantly correlated with the score assigned to sparing of vasopressin or VIP after SCN microlesions $(p>$ 0.05 in both cases). As with behavioral responses, rhythms in body temperature were significantly correlated with the score for the amount of CalB remaining $(p<0.05)$. Rhythms in heart rate exhibited a similar trend $(p=0.064)$. Vasopressin and VIP scores were not significantly correlated with the amplitude of either body temperature or heart rate rhythms $(p>0.05$ in both cases).

\section{Discussion}

The present study revealed that the CalB subregion of the hamster $\mathrm{SCN}$ is necessary for the expression of circadian rhythmicity in all physiological and behavioral rhythms measured. The results support the hypothesis that the CalB subregion is necessary for SCN function and all resulting rhythms and argues against the notion that distinct subregions of the SCN serve different rhythmic responses. In the present investigation, small lesions that ablated the CalB subregion, while sparing as much as $\sim 55 \%$ of the remaining SCN, abolished all behavioral (locomotor, drinking, gnawing), physiological (heart rate, body temperature), and endocrine (melatonin, cortisol) circadian rhythms measured. In contrast, after lesions of similar or even greater size that spared a portion of the CalB region of the SCN, circadian rhythms in behavior or physiology were sustained. Together, the present results provide evidence for an important SCN network in which a subregion marked by CalB is necessary for the maintenance of circadian rhythmicity.

As mentioned previously, the CalB region of the SCN is not endogenously rhythmic in Per1, Per2, and Per3 expression (Hamada et al., 2001), giving rise to the question of the function served by these cells. CalB cells receive direct retinal input, although retinal input is not restricted to this subregion (Bryant et al., 2000; Silver et al., 1996b). FOS, Per1, and Per2 are light in- 

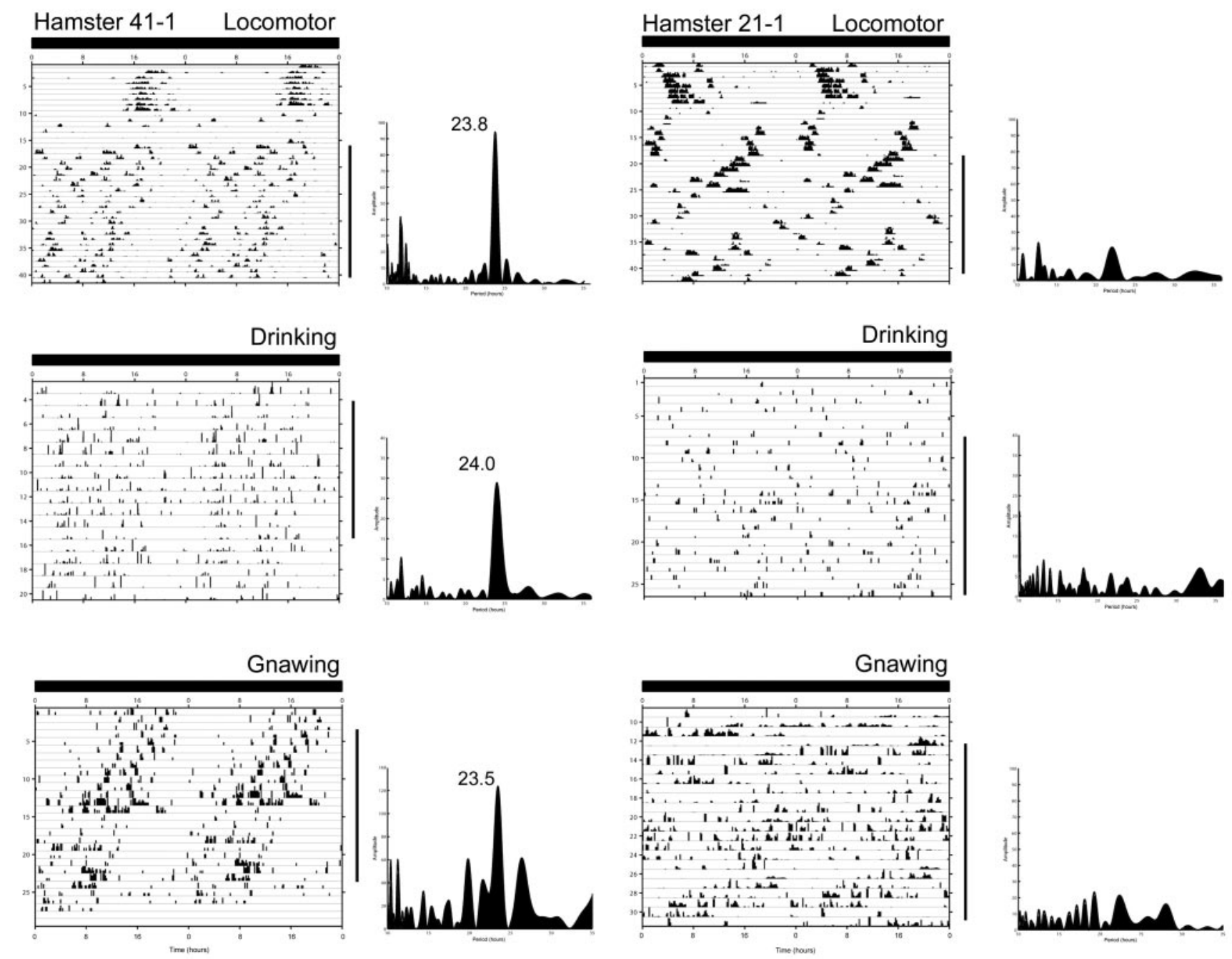

Figure 2. Actograms depicting locomotor (top), drinking (middle), and gnawing (bottom) behavior of animals housed in DD. Hamster 41-1 (left) had an SCN lesion that spared the CalB subregion unilaterally (see photomicrographs in Fig. 3). In contrast, hamster 21-4 (right) had an SCN lesion abolishing the CalB subregion bilaterally (see photomicrographs in Fig. 3). Spectral analyses are presented to the right of each actogram. Black vertical bars to the right of actograms indicate the days on which the analyses were performed.

duced in this SCN region (Silver et al., 1996b; Hamada et al., 2001). Thus, this subregion may be important for receiving and transducing environmental light information to synchronize the $\mathrm{SCN}$ to environmental time. Although CalB neurons do not express circadian rhythms in firing rate (Jobst and Allen, 2002), these cells do exhibit a circadian rhythm in the subcellular localization of CalB (Hamada et al., 2003). This finding suggests that either daily rhythms in the subcellular localization of CalB are driven by feedback from pacemaker cells in the SCN, or that this rhythm is generated by an unidentified cellular feedback loop independent of identified clock genes. The present study suggests a central role for this SCN subregion in the maintenance of circadian function. Whether or not CalB-ir cells specifically or other cell phenotypes in this subregion are required to maintain rhythmic function requires additional investigation.

The fact that the CalB region of the SCN is required for rhythmicity in physiology and behavior, but itself is not rhythmic in terms of clock gene expression, is perplexing. One potential resolution of this enigma is provided by the model of Antle et al. (2003). According to this model, the functional oscillatory SCN network is composed of nonrhythmic retinorecipient cells termed "gate cells" that are necessary to coordinate the population-wide rhythmicity of independent cellular oscillators. In turn, when these coordinated oscillators reach a threshold of synchronized activity, they produce a feedback signal to regulate the activity of these nonrhythmic gate cells. This model accounts for the role of cells in the CalB subregion in regulating rhythmicity, although these cells do not express endogenous rhythms in clock genes. This model further predicts that communication from pacemaker cells in the shell is necessary to close the network feedback loop required to maintain SCN tissue-level rhythmicity. This view is supported in empirical studies in which the SCN were separated by a surgical knife cut in the horizontal plane to isolate the top one-third of the SCN from the bottom two-thirds (Yamaguchi et al., 2004). After transection, individual cells in the dorsomedial subdivision maintained rhythmicity, but the synchrony among cells was lost (Yamaguchi et al., 2004).

One requirement of the organization described above is that cells in the CalB subregion must communicate with oscillator cells. Several lines of evidence point to a neural means of communication. First, fibers exiting the CalB subregion proceed dorsomedially to the SCN shell (Fig. 9), and double-label immunocytochemistry revealed that CalB cells in hamster SCN project to the vasopressin-rich region of the SCN (LeSauter et al., 2002). 
CalB cells also contact both gastrinreleasing peptide and VIP cells (LeSauter et al., 2002), both of which innervate the vasopressin region of the SCN, suggesting an additional means of multisynaptic communication. Interestingly, the SCN shell does not appear to project back to the SCN core in rats (Leak et al., 1999 ) or the CalB subregion in hamsters (Kriegsfeld et al., 2004). Thus, feedback to the CalB region of the SCN may occur via unidentified non-neural means. Because all identified SCN targets project back to the SCN (Moga and Moore, 1997), rhythmic information may also be communicated back to the CalB subregion from SCN targets. Whether or not this indirect communication exists, as well as its potential role in modulating rhythmic function, requires additional study.

The functional organization suggested by the present findings may be a common feature of SCN organization in mammals more generally. For example, recordings from rat SCN in culture demonstrate that, although $87 \%$ of shell neurons are rhythmic in neural firing, only $62 \%$ of ventral neurons recorded are rhythmic (Nakamura et al., 2001). Likewise, both laboratory rats and the diurnal Nile grass rat ( $A r$ vicanthis niloticus) have a SCN subregion marked by CalB (Arvanitogiannis et al., 2000; Mahoney et al., 2000). In rats (Miyake et al., 2000) and mice (Shigeyoshi et al., 1997), light-induced Per 1 expression is primarily restricted to the SCN core, whereas endogenously rhythmic Per1 mRNA is seen in the shell. In mice carrying a null mutation of the VIP receptor VPAC(2), rhythms in behavior and clock gene expression are disrupted (Harmar et al., 2002). Given that VIP neurons are located ventrally and project dorsally in mouse SCN, this finding suggests that ventral to dorsal communication is critical for the maintenance of circadian rhythmicity. Finally, we have recently shown that mice have a retinorecipient ventrolateral SCN subdivision marked by gastrin-releasing peptide-containing cells that is not rhythmic in terms of clock gene expression (Karatsoreos et al., 2004). Thus, although specific neurochemical markers may differ, the general structural and functional organization of the SCN may be conserved across mammalian species.

It could be argued that the abolition of rhythmic behavior and physiology after ablation of the CalB nucleus in the present study is attributable to damage to critical neural output pathways from the SCN. Several points argue against this possibil-
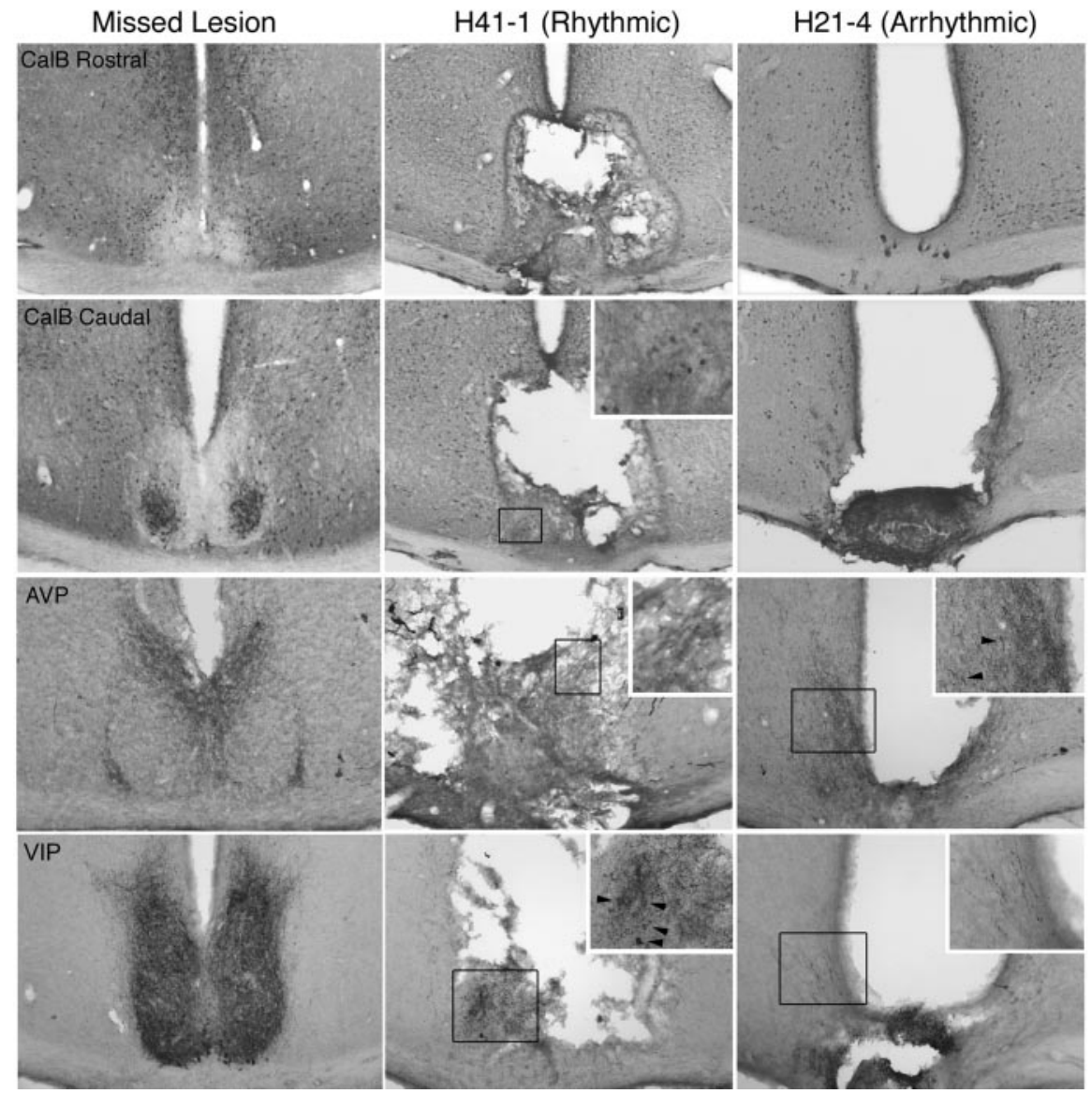

Figure 3. An example of three hamsters with lesions that either missed the SCN (left) or partial SCN lesions sparing (middle) or eliminating (right) the CaIB subregion. Hamster 41-1 has a comparatively large SCN lesion eliminating vasopression staining, while sparing CalB and VIP, and this animal remained rhythmic in all measures. In contrast, hamster 21-4 had a small SCN lesion eliminating CalB bilaterally, while sparing vasopressinergic and VIPergic cells and fibers. The phenotypes of these same animals are depicted in Figures 1 and $3-5$. The insets depict high-power photomicrographs of outlined regions, and arrows point to cell bodies.
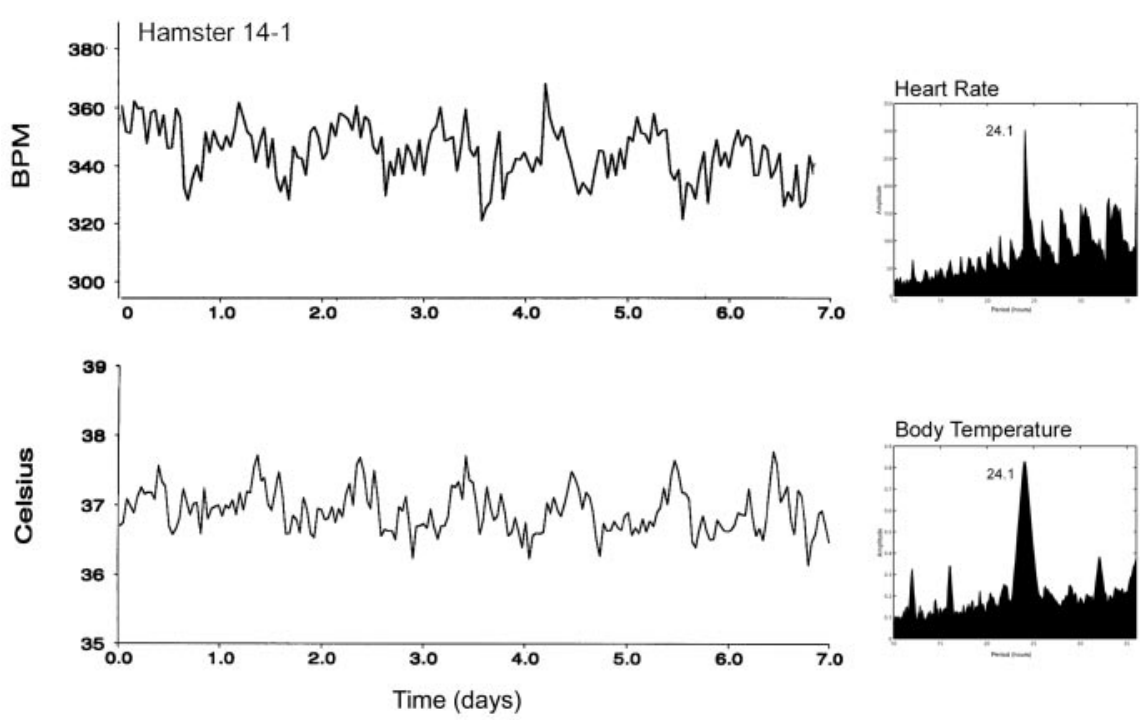

Figure 4. Records of heart rate and body temperature of a hamster (hamster 14-1) maintained in DD. This animal received a lesion that missed the SCN. Spectral analyses for the data presented are shown to the right of each record. 

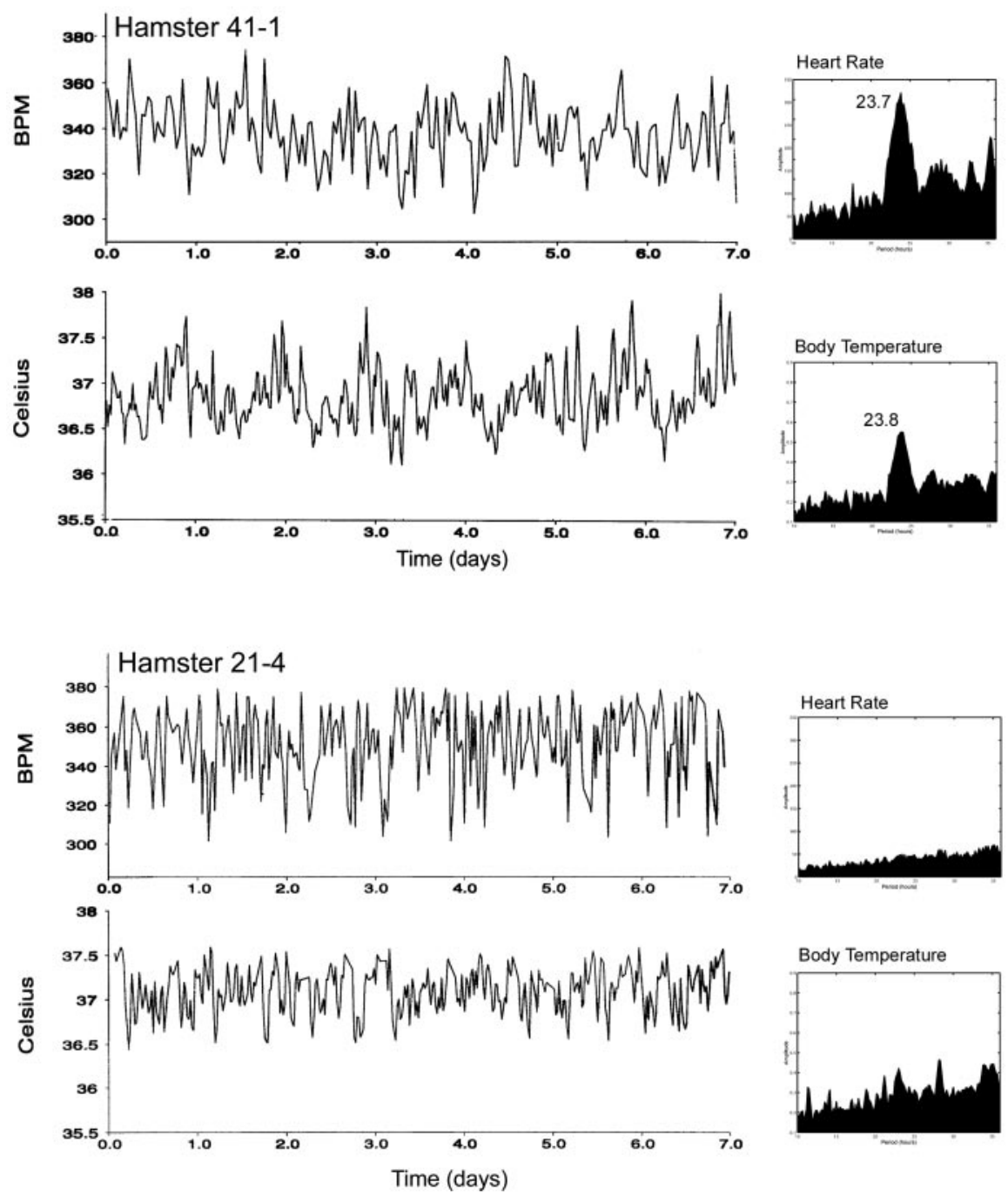

Figure 5. Records of heart rate and body temperature of animals maintained in DD that either had an SCN lesion that spared the CalB subregion unilaterally (top; see photomicrographs in Fig. 2) or abolished the CalB subregion bilaterally (bottom; see photomicrographs in Fig. 2). Spectral analyses for the data presented are shown to the right of each record. ity. First, behavioral rhythms can be supported by a diffusible signal not requiring neural output (Hakim et al., 1991; Silver et al., 1996a; Kramer et al., 2001; Cheng et al., 2002), suggesting that all behavioral rhythms would have been intact if SCN efferents were severed in the present study. In addition, vasopressin neurons in the SCN shell project to both the parasympathetic and sympathetic divisions of the autonomic nervous system in rats (Larsen et al., 1998; Larsen, 1999; Leak et al., 1999; Buijs et al., 2001), suggesting that some projections regulating rhythms in melatonin secretion and heart rate should be intact in animals with small CalB lesions in the present study. Finally, recent work from our own laboratory has shown that, in hamsters, projections to the preoptic area (responsible for body temperature regulation) and the paraventricular nucleus of the hypothalamus (regulation of cortisol and pre-autonomic regulation) arise predominantly from the SCN shell (Kriegsfeld et al., 2004). Together, these data suggest that lesions ablating the CalB region of the $\mathrm{SCN}$ abolish rhythmicty because of the destruction of a critical functional SCN compartment.

Severing SCN efferents results in loss of photoperiodic responses attributable to an abolition of melatonin rhythmicity (Nunez et al., 1985; for review, see Kriegsfeld et al., 2002) and likely accounts for the range of gonadal responses seen in animals with lesions sparing a portion of the CalB subnucleus. This likely accounts for the fact that one animal with sparing of the CalB subregion in the present study exhibited a loss of neuroendocrine rhythms, whereas all other rhythmic measures remained intact. It is unclear how to account for one animal with normal neuroendocrine rhythms in which the CalB subregion was ablated.

Although the notion of core and shell subdivisions of the rodent SCN have been a useful classification for investigating SCN topographic organization (Moore, 1996; Leak et al., 1999; Abrahamson and Moore, 2001; Moore et al., 2002), the CalB subregion of the hamster SCN cannot be readily mapped onto this dichotomy. Shell neurons are smaller than core neurons with few dendritic arbors. Core neurons receive retinal input and project more extensively within the SCN (Moore, 1996). Whereas CalB

Table 2. Summary of melatonin and cortisol rhythms in animals bearing partial SCN lesions

\begin{tabular}{llllll}
\hline Number & AVP & VIP & CalB & Melatonin & Cortisol \\
\hline 6 & + & + & - & - & - \\
1 & - & + & + & - & + \\
1 & - & + & - & + & + \\
4 & + & + & + & + & + \\
1 & + & + & + & - & - \\
\hline
\end{tabular}

Figure 6. ETV (in cubic millimeters) of hamsters held in DD for 8-10 weeks. Each point depicts the ETV of individual hamsters. Animals received a lesion that missed the $\mathrm{SCN}$ or either spared or abolished the CalB subregion of this nucleus.
+ , Presence of peptide or endocrine rhythm; - , absence of peptide or endocrine rhythm; AVP, arginine vasopressin. Each group represents a subset of animals from Table 1. 


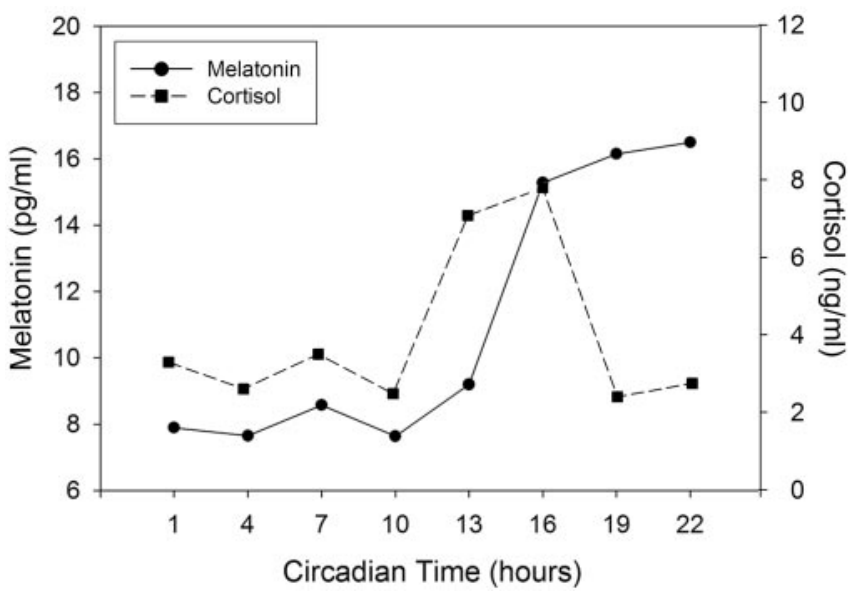

Figure 7. Serum cortisol and melatonin concentrations over the course of the day of an unlesioned control hamster implanted with a jugular catheter. The hamster was maintained in DD during the collection of blood samples.
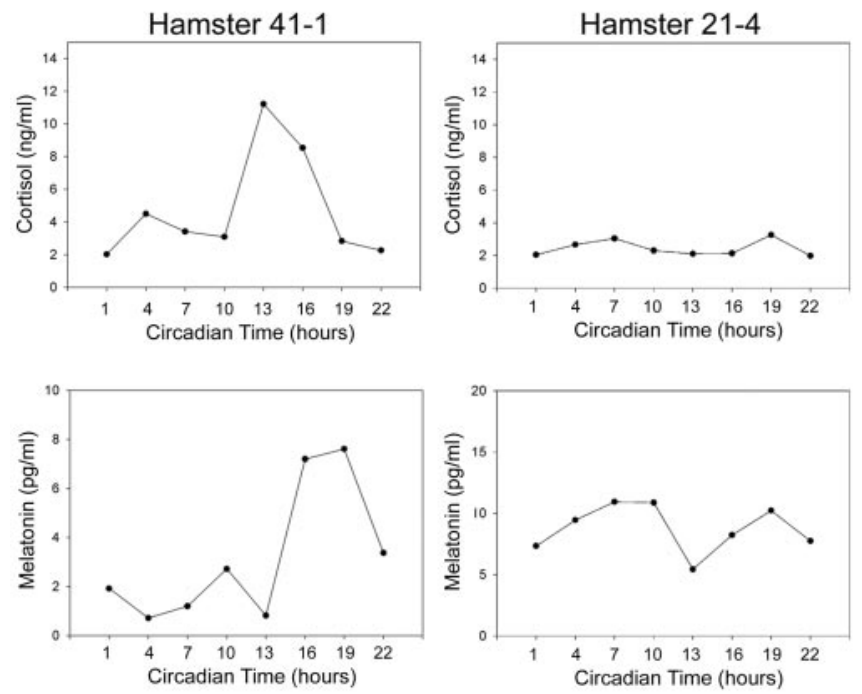

Figure 8. Serum cortisol (top) and melatonin (bottom) concentrations over the course of the day of animals receiving an $\mathrm{SCN}$ lesion either eliminating vasopression staining, while sparing CalB and VIP (hamster 41-1; left), or eliminating CalB bilaterally, while sparing vasopressinergic and VIPergic cells and fibers (hamster 21-4; right). Hamsters were maintained in DD during the collection of blood samples. Because there are not reference time points for arrhythmic animals held in $\mathrm{DD}$, precluding averaging group data, hormone data from individual animals are shown.

Table 3. Correlation between the magnitude of circadian measures and the amount of each peptide remaining after lesions in which rhythmicity was maintained

\begin{tabular}{llll}
\hline & AVP & VIP & CalB \\
\hline Wheel running & 0.371 & 0.433 & $0.666^{*}$ \\
Drinking & 0.436 & 0.426 & $0.743^{*}$ \\
Gnawing & 0.195 & 0.391 & $0.630^{*}$ \\
Body temperature & 0.298 & 0.299 & $0.604^{* *}$ \\
Heart rate & 0.302 & 0.379 & 0.574 \\
\hline
\end{tabular}

AVP, Arginine vasopressin. ${ }^{*} p<0.01 ;{ }^{* *} p<0.05$.

neurons in hamsters receive retinal input (Silver et al., 1996b; Bryant et al., 2000), their size, dendritic arbors, and location do not fit neatly into the core criteria defined in rats. Because of this inability to classify the hamster SCN into core and shell, along with previous findings demonstrating putative functional subdi-

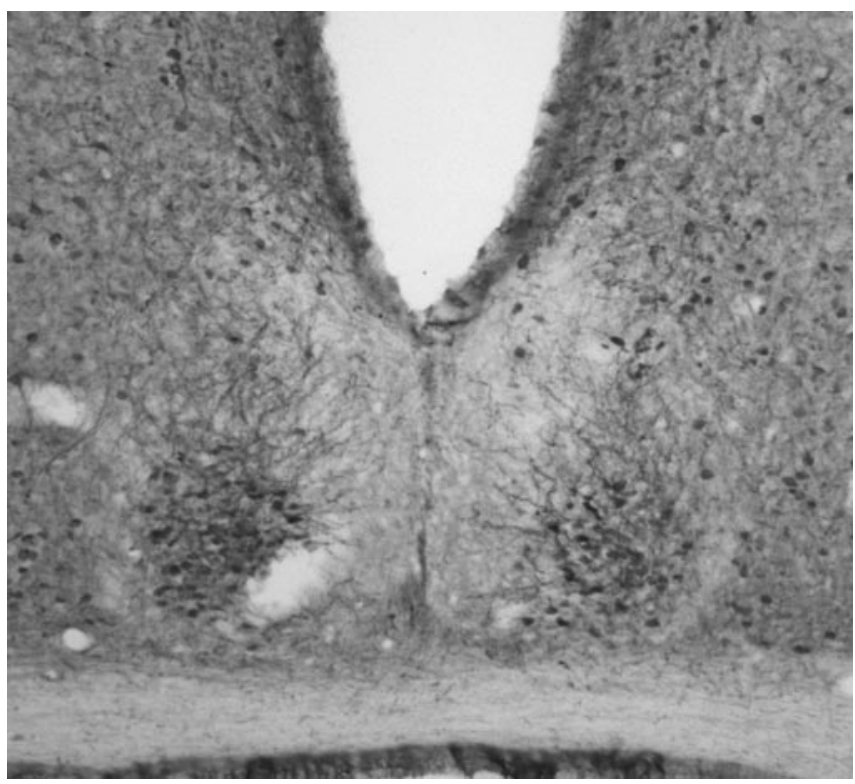

Figure 9. Coronal section through the caudal SCN stained immunohistochemically for CalB. Note that CalB fibers course dorsomedially toward the $\mathrm{SCN}$ shell.

visions in the hamster $\mathrm{SCN}$ are associated with peptidergic markers (Hamada et al., 2001, 2003), we sought to determine the functional significance of neurochemically defined SCN subdivisions. The present findings demonstrate that the subregion of the SCN in hamsters marked by CalB is a crucial component of the rhythm-generating network of the SCN. No other neurochemically defined subregion investigated was associated with disruptions in circadian function. These findings point to an important neural network requiring a tissue-level feedback loop comprising the CalB subregion necessary for sustaining circadian rhythms.

\section{References}

Abrahamson EE, Moore RY (2001) Suprachiasmatic nucleus in the mouse: retinal innervation, intrinsic organization and efferent projections. Brain Res 916:172-191.

Antle MC, Foley DK, Foley NC, Silver R (2003) Gates and oscillators: a network model of the brain clock. J Biol Rhythms 18:339-350.

Arvanitogiannis A, Robinson B, Beaule C, Amir S (2000) Calbindin-D28k immunoreactivity in the suprachiasmatic nucleus and the circadian response to constant light in the rat. Neuroscience 99:397-401.

Bryant DN, LeSauter J, Silver R, Romero MT (2000) Retinal innervation of calbindin-D28K cells in the hamster suprachiasmatic nucleus: ultrastructural characterization. J Biol Rhythms 15:103-111.

Buijs RM, Chun SJ, Niijima A, Romijn HJ, Nagai K (2001) Parasympathetic and sympathetic control of the pancreas: a role for the suprachiasmatic nucleus and other hypothalamic centers that are involved in the regulation of food intake. J Comp Neurol 431:405-423.

Cheng MY, Bullock CM, Li C, Lee AG, Bermak JC, Belluzzi J, Weaver DR, Leslie FM, Zhou QY (2002) Prokineticin 2 transmits the behavioural circadian rhythm of the suprachiasmatic nucleus. Nature 417:405-410.

Davis FC, Gorski RA (1988) Development of hamster circadian rhythms: role of the maternal suprachiasmatic nucleus. J Comp Physiol [A] 162:601-610.

Hakim H, DeBernardo AP, Silver R (1991) Circadian locomotor rhythms, but not photoperiodic responses, survive surgical isolation of the SCN in hamsters. J Biol Rhythms 6:97-113.

Hamada T, LeSauter J, Venuti JM, Silver R (2001) Expression of Period genes: rhythmic and nonrhythmic compartments of the suprachiasmatic nucleus pacemaker. J Neurosci 21:7742-7750.

Hamada T, LeSauter J, Lokshin M, Romero MT, Yan L, Venuti JM, Silver R (2003) Calbindin influences response to photic input in suprachiasmatic nucleus. J Neurosci 23:8820-8826.

Harmar AJ, Marston HM, Shen S, Spratt C, West KM, Sheward WJ, Morrison 
CF, Dorin JR, Piggins HD, Reubi JC, Kelly JS, Maywood ES, Hastings MH (2002) The VPAC(2) receptor is essential for circadian function in the mouse suprachiasmatic nuclei. Cell 109:497-508.

Harrington ME, Rahmani T, Lee CA (1993) Effects of damage to SCN neurons and efferent pathways on circadian activity rhythms of hamsters. Brain Res Bull 30:655-669.

Jobst EE, Allen CN (2002) Calbindin neurons in the hamster suprachiasmatic nucleus do not exhibit a circadian variation in spontaneous firing rate. Eur J Neurosci 16:2469-2474.

Karatsoreos I, Yan L, LeSauter J, Silver R (2004) Phenotype matters: Identification of light responsive cells in the mouse SCN. J Neurosci 24:68-75.

Kramer A, Yang FC, Snodgrass P, Li X, Scammell TE, Davis FC, Weitz CJ (2001) Regulation of daily locomotor activity and sleep by hypothalamic EGF receptor signaling. Science 294:2511-2515.

Kriegsfeld LJ, LeSauter JL, Hamada T, Pitts SM, Silver R (2002) Circadian rhythms in the endocrine system. In: Hormones, brain, and behavior (Pfaff DW, Arnold AP, Etgen AM, Fahrbach SE, Rubin RT, eds). New York: Academic.

Kriegsfeld LJ, Leak R, LeSauter J, Yackulic C, Silver R (2004) Organization of suprachiasmatic nucleus projections in Syrian hamsters (Mesocricetus auratus): an anterograde and retrograde analysis. J Comp Neurol 468:371-379.

Larsen PJ (1999) Tracing autonomic innervation of the rat pineal gland using viral transneuronal tracing. Microsc Res Tech 46:296-304.

Larsen PJ, Enquist LW, Card JP (1998) Characterization of the multisynaptic neuronal control of the rat pineal gland using viral transneuronal tracing. Eur J Neurosci 10:128-145.

Leak RK, Card JP, Moore RY (1999) Suprachiasmatic pacemaker organization analyzed by viral transynaptic transport. Brain Res 819:23-32.

Lehman MN, Silver R, Gladstone WR, Kahn RM, Gibson M, Bittman EL (1987) Circadian rhythmicity restored by neural transplant. Immunocytochemical characterization of the graft and its integration with the host brain. J Neurosci 7:1626-1638.

LeSauter J, Silver R (1994) Suprachiasmatic nucleus lesions abolish and fetal grafts restore circadian gnawing rhythms in hamsters. Restorative Neurol Neurosci 6:135-143.

LeSauter J, Silver R (1999) Localization of a suprachiasmatic nucleus subregion regulating locomotor rhythmicity. J Neurosci 19:5574-5585.

LeSauter J, Kriegsfeld LJ, Hon J, Silver R (2002) Calbindin-D(28K) cells selectively contact intra-SCN neurons. Neuroscience 111:575-585.

Mahoney MM, Nunez AA, Smale L (2000) Calbindin and Fos within the suprachiasmatic nucleus and the adjacent hypothalamus of Arvicanthis niloticus and Rattus norvegicus. Neuroscience 99:565-575.

Miyake S, Sumi Y, Yan L, Takekida S, Fukuyama T, Ishida Y, Yamaguchi S, Yagita K, Okamura H (2000) Phase-dependent responses of Per1 and Per2 genes to a light-stimulus in the suprachiasmatic nucleus of the rat. Neurosci Lett 294:41-44.
Moga MM, Moore RY (1997) Organization of neural inputs to the suprachiasmatic nucleus in the rat. J Comp Neurol 389:508-534.

Moore RY (1996) Entrainment pathways and the functional organization of the circadian system. Prog Brain Res 111:103-119.

Moore RY, Eichler VB (1972) Loss of a circadian adrenal corticosterone rhythm following suprachiasmatic lesions in the rat. Brain Res 42:201-206.

Moore RY, Speh JC, Leak RK (2002) Suprachiasmatic nucleus organization. Cell Tissue Res 309:89-98.

Nakamura W, Honma S, Shirakawa T, Honma K (2001) Regional pacemakers composed of multiple oscillator neurons in the rat suprachiasmatic nucleus. Eur J Neurosci 14:666-674.

Nunez AA, Brown MH, Youngstrom TG (1985) Hypothalamic circuits involved in the regulation of seasonal and circadian rhythms in male golden hamsters. Brain Res Bull 15:149-153.

Okamura H, Yamaguchi S, Yagita K (2002) Molecular machinery of the circadian clock in mammals. Cell Tissue Res 309:47-56.

Panda S, Hogenesch JB, Kay SA (2002) Circadian rhythms from flies to human. Nature 417:329-335.

Ralph MR, Foster RG, Davis FC, Menaker M (1990) Transplanted suprachiasmatic nucleus determines circadian period. Science 247:975-978.

Rusak B (1977) The role of the suprachiasmatic nuclei in the generation of circadian rhythms in the golden hamster, Mesocricetus auratus. J Comp Physiol 118:145-164.

Shigeyoshi Y, Taguchi K, Yamamoto S, Takekida S, Yan L, Tei H, Moriya T, Shibata S, Loros JJ, Dunlap JC, Okamura H (1997) Light-induced resetting of a mammalian circadian clock is associated with rapid induction of the mPer1 transcript. Cell 91:1043-1053.

Silver R, LeSauter J, Tresco PA, Lehman MN (1996a) A diffusible coupling signal from the transplanted suprachiasmatic nucleus controlling circadian locomotor rhythms. Nature 382:810-813.

Silver R, Romero MT, Besmer HR, Leak R, Nunez JM, LeSauter J (1996b) Calbindin-D28K cells in the hamster SCN express light-induced Fos. NeuroReport 7:1224-1228.

Stephan FK, Zucker I (1972) Rat drinking rhythms: central visual pathways and endocrine factors mediating responsiveness to environmental illumination. Physiol Behav 8:315-326.

Van den Pol AN, Powley T (1979) A fine-grained anatomical analysis of the role of the rat suprachiasmatic nucleus in circadian rhythms of feeding and drinking. Brain Res 160:307-326.

van den Pol AN, Tsujimoto KL (1985) Neurotransmitters of the hypothalamic suprachiasmatic nucleus: immunocytochemical analysis of 25 neuronal antigens. Neuroscience 15:1049-1086.

Welsh DK, Logothetis DE, Meister M, Reppert SM (1995) Individual neurons dissociated from rat suprachiasmatic nucleus express independently phased circadian firing rhythms. Neuron 14:697-706.

Yamaguchi S, Isejima H, Matsuo T, Okura R, Yagita K, Kobayashi M, Okamura H (2003) Synchronization of cellular clocks in the suprachiasmatic nucleus. Science 302:1408-1412. 